\title{
Effect of Split Gate Size on the Electrostatic Potential and 0.7 Anomaly within Quantum Wires on a Modulation-Doped GaAs/AIGaAs Heterostructure
}

\author{
L. W. Smith, ${ }^{1, *}$ H. Al-Taie, ${ }^{1,2}$ A. A. J. Lesage, ${ }^{1}$ K. J. Thomas, ${ }^{3, \dagger}$ F. Sfigakis, ${ }^{1}$ P. See, ${ }^{4}$ J. P. Griffiths, ${ }^{1}$ I. Farrer, ${ }^{1,}$ \\ G. A. C. Jones, ${ }^{1}$ D. A. Ritchie, ${ }^{1}$ M. J. Kelly, ${ }^{1,2}$ and C. G. Smith ${ }^{1}$ \\ ${ }^{1}$ Cavendish Laboratory, Department of Physics, University of Cambridge, \\ J. J. Thomson Avenue, Cambridge CB3 OHE, United Kingdom \\ ${ }^{2}$ Centre for Advanced Photonics and Electronics, Electrical Engineering Division, \\ Department of Engineering, $9 \mathrm{~J}$. J. Thomson Avenue, University of Cambridge, \\ Cambridge CB3 OFA, United Kingdom \\ ${ }^{3}$ Department of Electronic and Electrical Engineering, University College London, Torrington Place, \\ London WC1E 7JE, United Kingdom \\ ${ }^{4}$ National Physical Laboratory, Hampton Road, Teddington, Middlesex TW11 OLW, United Kingdom \\ (Received 12 August 2015; revised manuscript received 13 February 2016; published 25 April 2016)
}

\begin{abstract}
We study 95 split gates of different size on a single chip using a multiplexing technique. Each split gate defines a one-dimensional channel on a modulation-doped GaAs/AlGaAs heterostructure, through which the conductance is quantized. The yield of devices showing good quantization decreases rapidly as the length of the split gates increases. However, for the subset of devices showing good quantization, there is no correlation between the electrostatic length of the one-dimensional channel (estimated using a saddle-point model) and the gate length. The variation in electrostatic length and the one-dimensional subband spacing for devices of the same gate length exceeds the variation in the average values between devices of different lengths. There is a clear correlation between the curvature of the potential barrier in the transport direction and the strength of the " 0.7 anomaly": the conductance value of the 0.7 anomaly reduces as the barrier curvature becomes shallower. These results highlight the key role of the electrostatic environment in onedimensional systems. Even in devices with clean conductance plateaus, random fluctuations in the background potential are crucial in determining the potential landscape in the active device area such that nominally identical gate structures have different characteristics.
\end{abstract}

DOI: 10.1103/PhysRevApplied.5.044015

\section{INTRODUCTION}

Low-dimensional devices which exhibit quantummechanical effects are routinely created using nanostructure gates on modulation-doped heterostructures. Often, devices with similar gate designs can display very different characteristics at cryogenic temperatures due to unpredictable local variations in the electrostatic landscape. In this paper, we investigate the impact of gate size on two important quantum properties of split-gate devices: the conductance quantization [1,2] and the occurrence of the 0.7 anomaly [3]. Experimental and theoretical techniques are used to determine the lateral and longitudinal potential

\footnotetext{
*Corresponding author.

luke.smith@wisc.edu

Present address: Department of Physics, University of WisconsinMadison, Madison, WI 53706, USA.

Present address: Department of Physics, Central University of Kerala, Riverside Transit Campus, Kasaragod 671 314, Kerala, India.

${ }^{*}$ Present address: Department of Electronic \& Electrical Engineering, University of Sheffield, Sheffield S1 3JD, United Kingdom.
}

profile in the one-dimensional (1D) channel. We find the 0.7 anomaly is governed by the electrostatic potential, regardless of gate size. However, fluctuations in the background potential due to ionized dopants often overwhelm the effect of changes in gate size in defining the potential landscape in the 1D channel.

The split gate is the simplest mesoscopic device that can be used to study how device behavior is affected by gate size. The conductance through a split gate [4] is quantized in multiples of $G_{0}=2 e^{2} / h$ as a function of the voltage applied to the gates $[1,2]$ due to the formation of $1 \mathrm{D}$ subbands. For an ideal 1D conductor, this quantization occurs as long as the transport remains ballistic. The effect of split-gate size can be investigated either by varying the lithographic dimensions [5-8] or by fabricating several split gates in close proximity which act in series to modify the potential of a single 1D channel [9-12]. So far, it has been shown that the split-gate voltage $\left(V_{\mathrm{SG}}\right)$ at which the conductance through the 1D channel is pinched off occurs closer to zero for longer and narrower devices $[5,6]$. Additionally, the quality of conductance quantization degrades as the gate length increases $[8,13]$. This latter effect is related to the higher probability of encountering an 
TABLE I. Number of devices of each width and length.

\begin{tabular}{lcc}
\hline \hline Devices measured & Width $(\mu \mathrm{m})$ & Length $(\mu \mathrm{m})$ \\
\hline 15 & 0.4 & 0.4 \\
14 & 0.4 & 0.7 \\
15 & 0.4 & 1.0 \\
6 & 0.4 & 1.3 \\
15 & 0.6 & 0.4 \\
15 & 0.6 & 0.7 \\
15 & 0.6 & 1.0 \\
\hline \hline
\end{tabular}

impurity in the channel with longer or wider split gates and fluctuations in the background disorder potential which modify the potential landscape in the channel area.

Some studies of the effect of split-gate size have focused on the 0.7 anomaly $[6,7,10]$, a conductance feature which occurs near $0.7 G_{0}[3,14,15]$, which arises from enhanced electron interactions at low conductance. A reduction in the conductance of the 0.7 anomaly for longer split gates has been reported [7]. Another study using split gates in series has shown periodic modulations in the value of the 0.7 anomaly as a function of $1 \mathrm{D}$ channel length [10]. The origin of the 0.7 anomaly is currently debated; the theories proposed for its occurrence include spontaneous spin polarization [3,16], the Kondo effect [10,17-19], Wigner crystallization $[20,21]$, and inelastic scattering plus the local enhancement (smeared van Hove singularity) of the $1 \mathrm{D}$ density of states $[22,23]$.

We use a multiplexing scheme [24-29] to measure 95 split gates of seven different sizes on a GaAs/AlGaAs modulation-doped heterostructure. We study the impact of split-gate dimensions using data obtained from a large number of devices fabricated on a single chip and measured during one cooldown. Multiple devices of each dimension are measured to provide statistical information. Table I summarizes how many devices of each length and width are measured.

Our technique allows us to systematically compare incremental changes in gate design. This is important in the context of device development, where it may be necessary to design a device with a specific set of operating parameters or find ways of improving a particular design. The traditional approach to nanostructure measurement requires many cooldowns to build up the statistical information that we obtain in a single cooldown.

\section{DETAILED OUTLINE}

The device and heterostructure used in this experiment are described in Sec. III. In Sec. IV, we compare the accuracy of conductance quantization between split gates of different dimensions and find a rapid reduction in the quality of quantization as the gate length increases. We obtain the average values of the pinch-off voltage and definition voltage as a function of gate length and width which show how the pinch-off voltage occurs closer to zero for longer and narrower split gates, as expected from electrostatics [30]. For longer gates, the voltage at which the 1D channel is defined also occurs closer to zero, and the $1 \mathrm{D}$ subband spacing reduces.

A key finding of this paper is that the background disorder potential is at least as important as the split-gate dimension in governing the potential landscape in the 1D channel (Sec. V). The impact of background disorder is shown in three separate ways. First, the spread in both the measured 1D subband spacing and the estimated 1D barrier curvature for split gates at fixed gate lengths exceeds the variations in the mean value of these parameters between devices of different lengths. Second, the strength of the coupling between the split gates and the 1D channel does not monotonically increase with device length. A monotonic increase is expected if one considers only the electric field generated by the gates themselves. Third, changes in the 1D barrier curvature from device to device, which indicates the length of the 1D channel, do not depend on the split-gate length.

In Sec. VI, we directly compare the 0.7 anomaly in devices of different gate lengths. This is possible since, for a noninteracting system, the shape of the conductance trace depends on $\hbar \omega_{x, 1}$. We use a technique developed in Ref. [28] to remove the trivial geometric dependence from the conductance data, leaving differences that are only due to electron interactions. Our data are consistent with Ref. [28] in that the 0.7 anomaly occurs at lower conductance values for devices with a shallower longitudinal barrier. However, the present data set is gathered from devices of different dimensions (the data in Ref. [28] are from lithographically identical gates). By obtaining the same result from devices with various dimensions, we show that the electrostatic length of the 1D channel is the decisive factor governing the conductance of the 0.7 anomaly.

\section{METHODS}

The sample is fabricated on a GaAs/AlGaAs heterostructure in which the 2DEG forms $90 \mathrm{~nm}$ below the surface of the wafer, separated from a 40-nm-wide Si-doped AlGaAs layer by a 40-nm spacer layer of undoped AlGaAs. The carrier density and mobility are $2.0 \times 10^{11} \mathrm{~cm}^{-2}$ and $3.0 \times 10^{6} \mathrm{~cm}^{2} \mathrm{~V}^{-1} \mathrm{~s}^{-1}$, respectively, determined by measuring a Hall bar on a nearby piece of the wafer. The electron mean free path is $22 \mu \mathrm{m}$.

The split gates are arranged in an array of total area $1.5 \times 1.95 \mathrm{~mm}^{2}$. A schematic diagram of a split gate is given in Fig. 1(a). The split gates are defined using electron-beam lithography and metalized by thermally evaporating $\mathrm{Ti} / \mathrm{Au}$. A two-terminal constant-voltage technique is used to measure the differential conductance through the split gates as a function of gate voltage, using an ac excitation voltage of $100 \mu \mathrm{V}$ at $17 \mathrm{~Hz}$. All measurements are carried out at $1.4 \mathrm{~K}$ and $B=0 \mathrm{~T}$. 

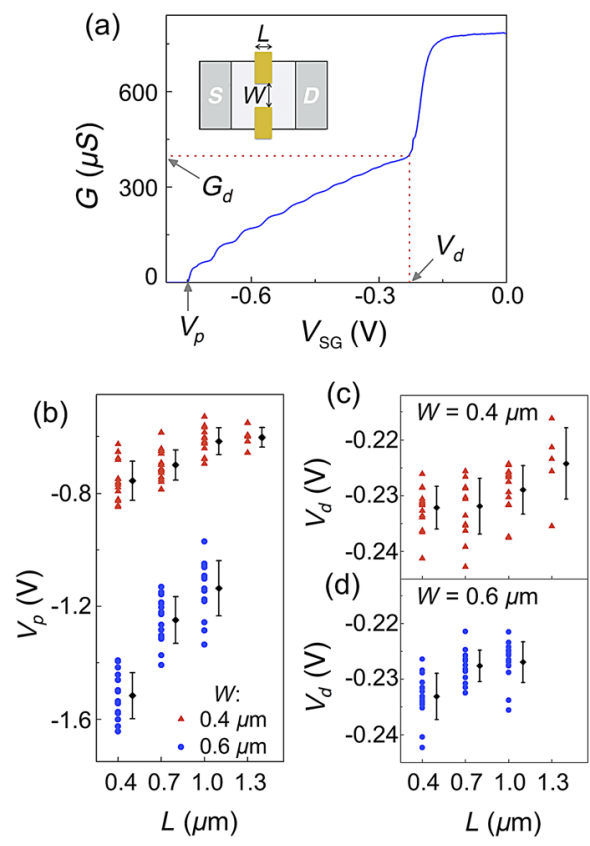

FIG. 1. (a) Typical trace of conductance $G$ as a function of the voltage applied to the split gate $V_{\mathrm{SG}}$. The vertical and horizontal dotted lines indicate the $1 \mathrm{D}$ definition voltage $\left(V_{d}\right)$ and conductance $\left(G_{d}\right)$, respectively. The left-hand arrow indicates pinchoff voltage $V_{p}$. The inset shows a schematic split-gate device with dimensions $L$ and $W$ labeled. Source and drain Ohmic contacts are marked $S$ and $D$, respectively. (b) Scatter plot of pinch-off voltage as a function of split-gate length $L$. The triangles (circles) represent data from devices of width $W=0.4(0.6) \mu \mathrm{m}$. The diamonds and error bars show the mean and standard deviation for each $L$, respectively, offset horizontally by $0.1 \mu \mathrm{m}$ for clarity. Panels (c) and (d) show $V_{d}$ as a function of $L$, for $W=0.4$ and $0.6 \mu \mathrm{m}$, respectively.

The device measured here is different from that used in our previous work on multiplexed split gates [24-26,28]. The earlier work used an array of identical split gates, where both the length and width were $0.4 \mu \mathrm{m}$. The multiplexed array measured here contains split gates of seven different length and width combinations and is fabricated on a higher-mobility heterostructure. This paper presents a self-contained story of the influence (or lack thereof) of the gate length on conductance in 1D devices. We investigate the effect of split-gate dimensions on device yield, 1D conductance properties, the electrostatic potential profile, and the 0.7 anomaly.

\section{CONDUCTANCE PROPERTIES AS A FUNCTION OF SPLIT-GATE SIZE}

\section{A. Electrical properties}

We begin by investigating the effect of split-gate length and width on the definition voltage $V_{d}$ and pinch-off voltage $V_{p}$. Figure 1(a) shows a typical plot of the conductance as a function of the voltage applied to the split gate $V_{\mathrm{SG}}$. A 1D channel is formed when the 2DEG beneath the gates is fully depleted, indicated by a sudden change in the gradient of the conductance trace [corresponding to voltage $V_{d}$ and conductance $G_{d}$ in Fig. 1(a)]. As $V_{\mathrm{SG}}$ decreases further, the conductance reduces (showing a series of plateaus) until the channel is completely pinched off (marked by $V_{p}$ ).

Figure 1(b) shows a scatter plot of $V_{p}$ against $L$, in which the triangles (circles) represent data for $W=0.4(0.6) \mu \mathrm{m}$. The diamonds or error bars show average values or standard deviation for each $L$. Both sets of data show $V_{p}$ becoming closer to zero with increasing $L[5,6]$. The trend is more pronounced for $W=0.6 \mu \mathrm{m}$, where $\left|V_{p}\right|$ is nearly double that of $W=0.4 \mu \mathrm{m}$. These trends arise from simple electrostatics, since for a given $V_{\mathrm{SG}}$, the electric field is stronger in the center of the channel for longer and narrower split gates [30]. An additional effect also occurs: a longer wire is more likely to be affected by fluctuations in the background disorder potential due to ionized donors. These fluctuations can modify the confining potential and, therefore, $V_{p}$. The larger spread in $V_{p}$ for $W=0.6 \mu \mathrm{m}$ may reflect the increased role of disorder. However, the spread as a percentage of the mean is similar for both $W$ 's.

Figures 1(c) and 1(d) show $V_{d}$ against $L$ for the $W=0.4$ and $0.6 \mu \mathrm{m}$ devices, respectively. In both cases, the magnitude of $V_{d}$ reduces as $L$ increases, which can be attributed to the higher electric field strength in the center of the channel for longer devices. For each $L$, the range and average values of $V_{d}$ are similar for both widths.

\section{B. Yield}

We investigate the role of disorder as a function of device length and width. The values of the conductance plateaus are used to define a yield criterion, since when disorder affects the transmission through a 1D channel, the conductance plateaus deviate from the expected values. For systematic analysis, the data are first corrected for series resistance $\left(R_{s}\right)$ using $R_{s}=1 / G$ at $V_{\mathrm{SG}}=0 \mathrm{~V}$ (i.e., the open-channel resistance). Two cases $(A$ and $B$ ) are considered. Case $A$ follows Ref. [24] and requires the first two conductance plateaus to occur within $\pm 0.1 G_{0}$. Case $B$ extends this to include the third plateau.

Figures 2(a) and 2(b) show the number of devices which pass the yield criterion as a function of $L$, for $W=0.4$ and $0.6 \mu \mathrm{m}$, respectively. The triangles and circles indicate the devices which passed for cases $A$ and $B$, respectively. Fewer devices show accurate conductance quantization as $W$ or $L$ increases $[8,13]$ due to the increased likelihood of encountering impurities in the 1D channel and greater variation in the background potential. Including the third plateau in the analysis gives a stricter yield criterion and leads to a lower yield.

The specific form of the relationship between yield and length in Fig. 2 is not clear: it is necessary to measure more than 15 devices of each $L$ to obtain this information. 


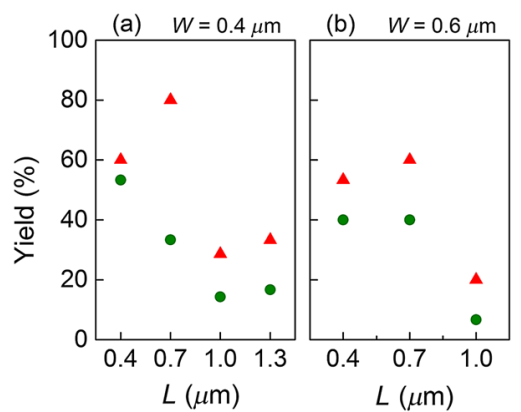

FIG. 2. Percentage of devices showing clean quantization as a function of split-gate length $L$. Panels (a) and (b) show data for widths $W=0.4$ and $0.6 \mu \mathrm{m}$, respectively. The triangles (circles) indicate devices for which the first and second (first, second, and third) plateaus occur within $\pm 0.1 G_{0}$, after correcting for series resistance.

Quantifying the exact nature of the correlation between yield and length is an interesting avenue for future research which may provide information about disorder correlation lengths or dominant disorder effects in the 1D channel.

\section{DEPENDENCE OF THE ELECTROSTATIC POTENTIAL LANDSCAPE ON GATE LENGTH}

Having characterized the length and width dependence of two electrical properties of the 1D conductance trace $\left(V_{p}\right.$ and $V_{d}$ ), we now consider the length dependence of the potential profile for a subset of devices. We first investigate the transverse confining potential by extracting the $1 \mathrm{D}$ subband spacing [31]. We then study the longitudinal potential by estimating the curvature of the barrier in the direction of electron transport [25]. The following measurements and analysis are performed using devices with $W=0.4 \mu \mathrm{m}$, since a higher percentage of split gates with $W=0.6 \mu \mathrm{m}$ were affected by disorder.

\section{A. dc bias spectroscopy}

dc bias spectroscopy is used to measure the 1D subband spacing [31] for 18 split gates. Figure 3(a) shows an example gray-scale plot of the transconductance $d G / d V_{\mathrm{SG}}$ as a function of source-drain bias $V_{\mathrm{SD}}$ and $V_{\mathrm{SG}}$ [dark (light) region corresponds to high (low) transconductance]. The data are corrected to remove all sources of series resistance such as Ohmic contact resistance (the method used is described in detail in the Supplemental Material of Ref. [32]).

As $\left|V_{\mathrm{SD}}\right|$ increases, dark regions representing peaks in the transconductance diverge into two (highlighted by the dashed lines), corresponding to the bottom of the $1 \mathrm{D}$ subband reaching either the source or drain chemical potential [33]. Two lines cross when $V_{\mathrm{SD}}$ is equal to the energy difference between consecutive 1D subbands, giving the $1 \mathrm{D}$ subband spacing $\Delta E_{n, n+1}$ ( $n$ is the subband index). For example, $\Delta E_{2,3}$ is marked in Fig. 3(a).
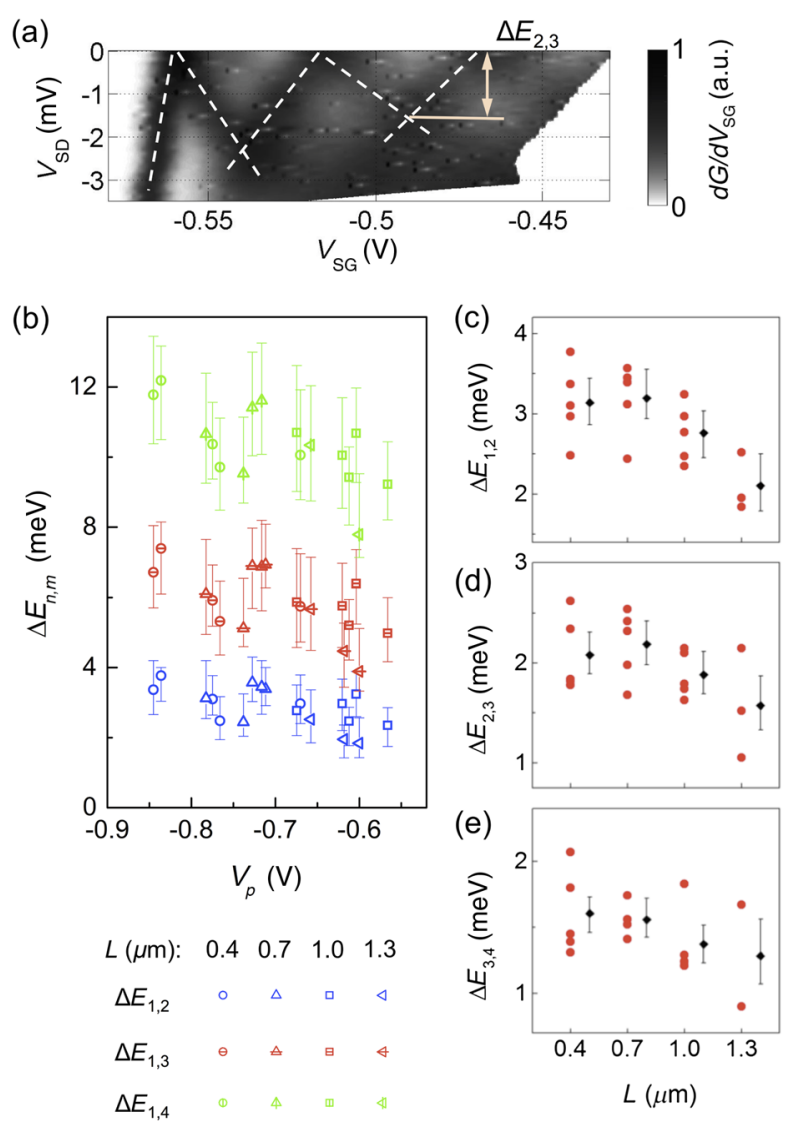

FIG. 3. (a) Gray-scale diagram of the transconductance $d G / d V_{\mathrm{SG}}$ as a function of $V_{\mathrm{SG}}$ and source-drain bias $V_{\mathrm{SD}}$, from a typical device with dimensions $L(W)=0.4$ (0.4). Black (white) regions correspond to high (low) transconductance, i.e., transitions between plateaus (the plateaus themselves). The 1D subband spacings are estimated by the crossings of peaks in the transconductance [31] highlighted by dashed lines. Subband spacing $\Delta E_{2,3}$ is labeled for illustrative purposes. (b) Cumulative $1 \mathrm{D}$ subband spacings $\Delta E_{n, m}$ as a function of pinch-off voltage $V_{p}$. The blue, red, and green symbols correspond to $\Delta E_{1,2}, \Delta E_{1,3}$, and $\Delta E_{1,4}$, respectively. Unique symbols represent devices of each size, as described in the legend. The error bounds show the cumulative error in the estimate. Data for $\Delta E_{1,3}$ and $\Delta E_{1,4}$ are offset vertically by 1 and $4 \mathrm{meV}$, respectively, for clarity. (c)-(e) Scatter plots of $\Delta E_{n, n+1}$ against $L$ for $n=1,2$, and 3 , respectively (to avoid confusion, the error bars on individual data points are not shown). The diamonds show the average for each $L$ (offset horizontally by $0.1 \mu \mathrm{m}$ for clarity), and error bounds indicate the average error.

Figure 3(b) shows cumulative 1D subband spacings $\Delta E_{n, m}$ as a function of $V_{p}$ for 18 devices. The spacing between the first and second $\left(\Delta E_{1,2}\right)$, first and third $\left(\Delta E_{1,3}\right)$, and first and fourth $\left(\Delta E_{1,4}\right)$ subbands is shown (unique symbols represent devices of different $L$ described in the legend). The data for $\Delta E_{1,3}$ and $\Delta E_{1,4}$ are vertically offset by 1 and $4 \mathrm{meV}$, respectively, for clarity.

In each case, $\Delta E_{n, m}$ reduces as $V_{p}$ becomes closer to zero. We plot the cumulative data to better accentuate this 
trend, although the spacing between consecutive subbands [shown in Figs. 3(c)-3(e)] all show a downward trend with $V_{p}$. The trend can be attributed to the weakening of the electric field at pinch-off for smaller $\left|V_{p}\right|$, since the confinement is shallower which leads to closer subband spacings.

Figures 3(c)-3(e) show individual 1D subband spacings as a function of $L$. Figures 3(c), 3(d), and 3(e) show $\Delta E_{1,2}$, $\Delta E_{2,3}$, and $\Delta E_{3,4}$, respectively. The diamonds represent average values for each $L$ (offset horizontally for clarity), and error bounds show the average error for each $L$ [34]. The average subband spacing reduces with $L$ in agreement with electrostatic modeling of a saddle-point potential [6,30]. However, the spread in $\Delta E_{n, n+1}$ for individual values of $L$ is larger than or similar to the average change in $\Delta E_{n, n+1}$ across all values of $L$. Since variations in device characteristics likely arise from fluctuations in the background potential, these data highlight the importance of unique electrostatic environment near each split gate. They suggest that background variations are as significant as the lithographic dimensions in governing the potential landscape in the device active area.

\section{B. Coupling between the split gate and the 1D channel}

The degree of coupling between the split gate and the 1D channel is given by lever arm $\alpha=\Delta E / e \Delta V_{\mathrm{SG}}$, where $\alpha=$ $\partial V_{\mathrm{SD}} / \partial V_{\mathrm{SG}}$ (estimated from dc bias spectroscopy measurements). Figure 4(a) shows $\alpha_{1}$ (the lever arm for the first subband), as a function of $L$ for 18 split gates. The diamonds indicate the average value offset horizontally for clarity. The error bounds indicate the average error [34].

The lever arm $\alpha_{1}$ almost doubles between $L=0.4$ and $0.7 \mu \mathrm{m}$. This trend is expected since $\alpha$ scales with $\Delta E$, which, for a given $\Delta V_{\mathrm{SG}}$, will be larger in the center of a channel formed by a longer gate. However, as $L$ increases further, $\alpha_{1}$ reduces, deviating from the expected trend. These data are discussed in more detail in Sec. V D. The same trend also occurs for subbands 2 and 3.

Figure 4(b) shows $\alpha_{1}$ as a function of $\Delta E_{1,2}$. Data from each length split gate are represented using different symbols described in the legend. Overall, no trend is apparent. Data from the same length devices appear to be grouped, and a weak positive correlation exists for the $L=0.4 \mu \mathrm{m}$ data. No correlation exists for longer devices, consistent with the increased effect of disorder.

\section{Curvature of the 1D potential barrier}

We now estimate the curvature of the potential barrier in the transport direction $\hbar \omega_{x}$ by assuming the confining potential is described by a saddle-point model [35]. We achieve this by fitting the measured data with a conductance calculated using the Landauer-Büttiker formalism $[25,28]$ simulating a system of noninteracting electrons traversing a saddle-point potential with transmission
- $L=0.4 \mu \mathrm{m} \cdot L=0.7 \mu \mathrm{m}$

، $L=1.0 \mu \mathrm{m}$ \& $L=1.3 \mu \mathrm{m}$
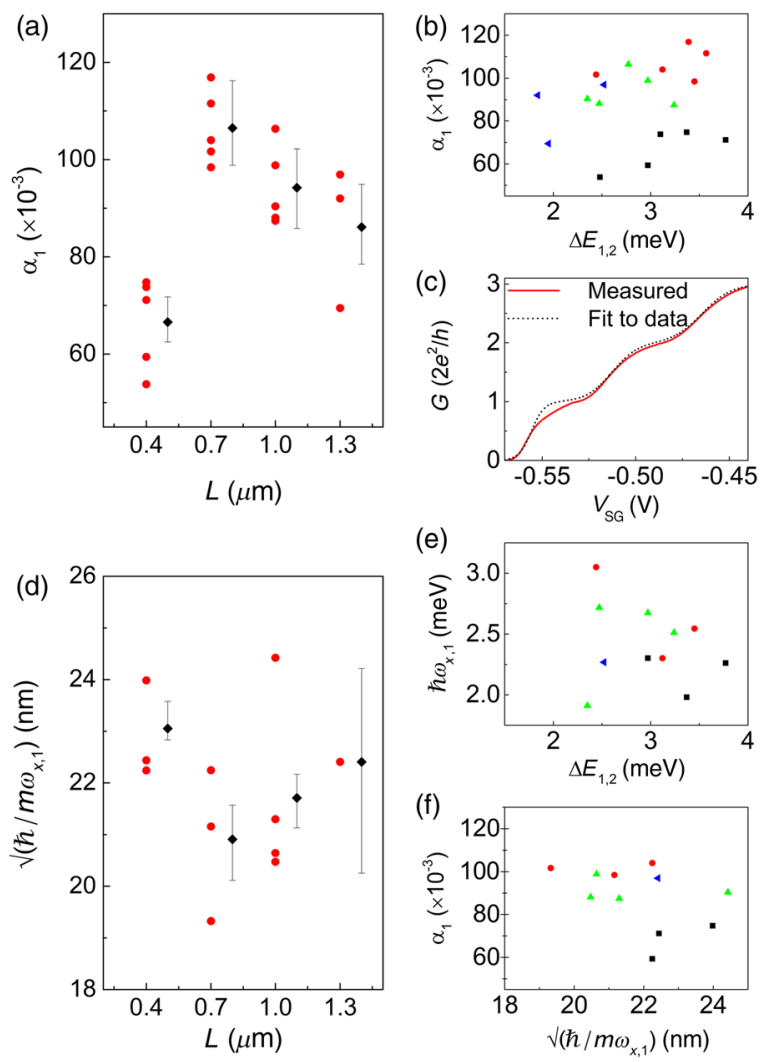

FIG. 4. (a),(b) Lever arm $\alpha_{1}$ as a function of lithographic length $L$ and $1 \mathrm{D}$ subband spacing $\Delta E_{1,2}$, respectively. The diamonds in panel (a) represent the mean for each $L$ (offset by $0.1 \mu \mathrm{m}$ for clarity), and the error bars indicate the average error. (c) Experimentally measured conductance $G$ as a function of split-gate voltage $V_{\mathrm{SG}}$, for an example device (solid line). The dashed line shows a fit to the data using a modified saddle-point model. The transition between plateaus gives an estimate of barrier curvature $\hbar \omega_{x, n}$. (d) Characteristic length of the potential barrier $\sqrt{\hbar / m^{*} \omega_{x, 1}}$ as a function of gate length $L$. The diamonds and error bars represent the average value and the average error, respectively (offset by $0.1 \mu \mathrm{m}$ for clarity). (e) Curvature of the potential barrier $\hbar \omega_{x, 1}$ as a function of $\Delta E_{1,2}$. (f) Lever arm $\alpha_{1}$ as a function of the characteristic length of the potential barrier. The different symbols in panels (b), (e), and (f) represent data for different length split gates as defined in the legend above panel (b).

probability $T_{n}=\left\{1+\exp \left[-2 \pi\left(E-E_{n}\right) / \hbar \omega_{x, n}\right]\right\}^{-1}$, where $E_{n}$ is the energy of the bottom of subband $n$. The saddle-point approximation can be used for the first few subbands, even for devices with a large length-to-width ratio, since $G$ is governed by transmission through the narrowest part of the channel.

Figure 4(c) shows the measured conductance (solid line) and the fit (dashed line) for an example device, as a function of $V_{\mathrm{SG}}$. The fit is achieved as follows: Transmission probability $T_{n}$ is calculated for the first three subbands individually as a function of $E$, using an initial 
input of $\hbar \omega_{x, n}=\Delta E_{n, n+1}$. Subband-dependent lever arms $\alpha_{n}$ measured for each device individually are used to convert $E$ to a voltage scale. A minimization routine then optimizes $\hbar \omega_{x, n}$ to find the best fit between the calculated and measured traces. The use of subband-dependent values of $\hbar \omega_{x, n}$ reflects how the barrier profile for higher subbands is modified by the increased presence of electrons. The sum of $T_{n}$ for $n=1,2$, and 3 gives the final trace shown by the dashed line in Fig. 4(c).

The transition between $G=0$ and $0.5 G_{0}$ is almost independent of temperature up to at least $T \approx 1.5 \mathrm{~K}$ [3]. Therefore, for $n=1$, the fit is performed with $T=0$, such that the calculated conductance $G_{n}=G_{0} T_{n}$. For higher subbands, a temperature dependence is observed experimentally; therefore, for $n=2$ and 3 , we calculate $G_{n}$ at $T=1.4 \mathrm{~K}$ using

$$
G_{n}=G_{0} \int d E\left(-\frac{\partial f}{\partial E}\right) T_{n}
$$

where $f$ is the Fermi-Dirac distribution. We find that using either $T=0$ or $T=1.4$ for $n=1$ does not affect the trends observed [28].

Good fits are obtained for 11 of the 18 split gates. For the other seven devices, conductance plateaus are weakened or suppressed due to strong disorder effects; therefore, these data are discarded in the following analysis. The saddlepoint model assumes a parabolic potential barrier, which has a characteristic length $l_{x}=\sqrt{\hbar / m^{*} \omega_{x, n}}$, where $m^{*}$ is the effective mass of the electrons. Barrier length $l_{x}$ is, therefore, the distance over which the potential changes by $\hbar \omega_{x} / 2$.

Figure 4(d) shows $l_{x, 1}$ as a function of $L$. The diamonds show the average $\bar{l}_{x}$ for each $L$, and error bounds indicate the average error offset horizontally for clarity. We expect the barrier length to scale with the gate length. However, our data show the opposite trend with $\bar{l}_{x}$ initially reducing for $L$ from 0.4 to $0.7 \mu \mathrm{m}$. This change is very small compared to the change of $L: \Delta \bar{l}_{x} \simeq 2.5 \mathrm{~nm}$ (a decrease of $11 \%$ ), as $L$ increases by $75 \%$. As $L$ increases further, $\bar{l}_{x}$ then increases slightly. For our devices, therefore, the splitgate length is not a good indicator of the length of the potential barrier.

For $L=0.4,0.7$, and $1 \mu \mathrm{m}$, the spread of $l_{x, 1}$ at fixed $L$ is larger than or similar to the overall change in average $l_{x, 1}$ as $L$ varies. This spread highlights the crucial importance of the background potential in determining the electrostatic landscape in the active device area, even between devices with nominally identical length. The importance of the background potential is further highlighted by Fig. 4(e), which shows $\hbar \omega_{x, 1}$ as a function of $\Delta E_{1,2}$. For a given $L$, there is no correlation between these parameters. The trends for subbands 2 and 3 are similar.

\section{Disorder potential fluctuations}

Our data indicate that the disorder potential is very important in determining the potential landscape in the 1D channel, and fluctuations in the disorder potential leads to deviations from the expected dependence on gate size. For example, the lever arm $\alpha$ depends only on split-gate length up to $L=0.7 \mu$ m [Fig. 4(a)], as discussed in Sec. V B. This may imply a correlation length of potential fluctuations close to this value. The correlation length may set an "effective" device length, leading to deviations from the expected trend for gates which would otherwise define a potential profile exceeding this length.

We now compare our results with calculations of the effect of potential fluctuations in the 2DEG on split-gate devices [36]. In Ref. [36], the quality of quantization was seen to degrade as a function of gate length (calculations were performed for $0.2-$ and $0.6-\mu \mathrm{m}$-long split gates, both $0.3 \mu \mathrm{m}$ wide). The quantization was also affected by the positioning of donors creating different distributions of the disorder potential.

The donor density for the heterostructure modeled in Ref. [36] is similar to ours, and the position of the donors was assumed to be random. The calculations are performed for a $\delta$-doped heterostructure with $N_{D}^{(2 \mathrm{D})}=4 \times 10^{12} \mathrm{~cm}^{-2}$, separated from the 2DEG by $42 \mathrm{~nm}$ of undoped AlGaAs. For comparison, our heterostructure contains a 40-nm Si-doped AlGaAs layer with a doping density $N_{D}^{(3 \mathrm{D})}=1.1 \times 10^{18} \mathrm{~cm}^{-3}$, separated from the 2DEG by $40 \mathrm{~nm}$ of undoped AlGaAs. We can estimate an effective 2D dopant density by assuming the doping layer is infinitesimally thick, which gives $N_{D}^{(2 \mathrm{D})}=4.4 \times 10^{12} \mathrm{~cm}^{-2}$, corresponding to a lateral spacing of less than $0.01 \mu \mathrm{m}$. The disorder correlation length given in Ref. [36] of around $0.2 \mu \mathrm{m}$ is not dissimilar to the value of $0.7 \mu \mathrm{m}$ beyond which $\alpha$ deviates from the expected gate-length dependence.

The lever arm $\alpha$ is determined from dc bias spectroscopy. As discussed in Ref. [37], the role of electron-electron interactions is important for measurements with a finite dc bias. Since electron interactions are enhanced at the top of the 1D potential barrier $[22,23,38]$, i.e., the interaction strength depends on the electrostatic profile, it is likely that variations in the potential between devices can significantly influence individual device behavior.

Figure 4(f) shows the dependence of $\alpha$ as a function of the characteristic barrier length $l_{x, 1}$. The data do not show a clear correlation [despite the apparent mirroring of data in Figs. 4(a) and 4(d)]. This highlights the sensitivity of device behavior to the exact potential environment.

Information on the length of fluctuations in the potential landscape can also be obtained from observations of Coulomb blockadelike resonances in conductance traces. These resonances can occur when the length of 1D channel is commensurate with the length scale of 
disorder fluctuations such that distortions of the potential profile form quantum dots in the 1D channel [39,40]. However, lower-temperature measurements are needed to clearly resolve these peaks in our devices.

Additional experiments can be devised to further investigate length scales of disorder fluctuations. For example, measuring identical split gates on a number of heterostructures with different thicknesses of undoped AlGaAs separating the $2 \mathrm{DEG}$ from the doped $\mathrm{AlGaAs}$ layer. As the 2DEG moves closer to the ionized impurities, the fluctuations in the background potential seen by the 2DEG will become sharper. The increased disorder typically manifests in a lower mobility for wafers with a smaller spacer spacing $[41,42]$. Greater disorder also leads to increased distortions in the 1D potential defined by split gates and degrade the quality of the conductance quantization, lowering the yield as defined in Sec. IV B.

So far, our discussion has focused on measurements on a modulation-doped GaAs/AlGaAs heterostructure. Another means of obtaining information on the role of disorder is to perform measurements on undoped heterostructures where the absence of $\mathrm{Si}$ donors results in a reduction of background disorder [43], leading to more reproducible behavior both between split-gate devices $[44,45]$ and after thermal cycling [46]. Measuring an array of devices on an undoped structure will also provide insight into the degree to which disorder affects nanostructures fabricated on undoped heterostructures [47].

\section{0.7 ANOMALY IN DIFFERENT LENGTH SPLIT GATES}

In this section, the 0.7 anomalies from devices of different gate lengths are compared. Following Bauer et al. [23], we refer to the conductance between $0.5 G_{0}$ and $G_{0}$ as the "subopen" regime. Additionally, we refer to experimentally measured (fitted) conductance data as $G_{e}\left(G_{f}\right)$.

Direct comparison of the 0.7 anomaly between devices is possible by removing the trivial-that which can be accounted for in a noninteracting scenario [35] dependence of the conductance transition between $G=0$ and $G_{0}$ on the barrier curvature. This is achieved by offsetting the conductance traces horizontally to align $G_{f} / G_{0}=0.5$ to $V_{\mathrm{SG}}=0$ and scaling each $V_{\mathrm{SG}}$ axis by $\alpha_{1} e / \hbar \omega_{x, 1}$ [28]. The differences in conductance that remain are only due to electron interactions. The strength of these interactions still depends on the barrier shape [22,23].

Figure 5(a) shows the fitted conductance $G_{f}$ for the first subband as a function of the scaled voltage axis $\kappa=\alpha_{1} e V_{\mathrm{SG}} / \hbar \omega_{x, 1}$. Data from all 11 split gates are plotted. The traces collapse onto a universal curve since these data are obtained using a noninteracting model. Figure 5(b) shows the corresponding $G_{e} / G_{0}$ data after applying the same scaling procedure. For $\kappa \leq 0$, the traces collapse onto a very similar curve. For $\kappa>0$, differences occur due to the (a)

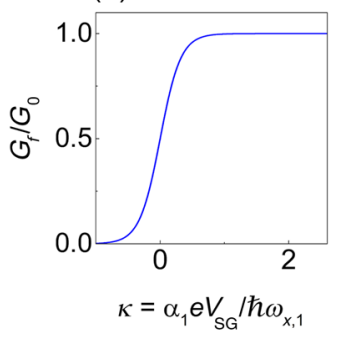

(c)

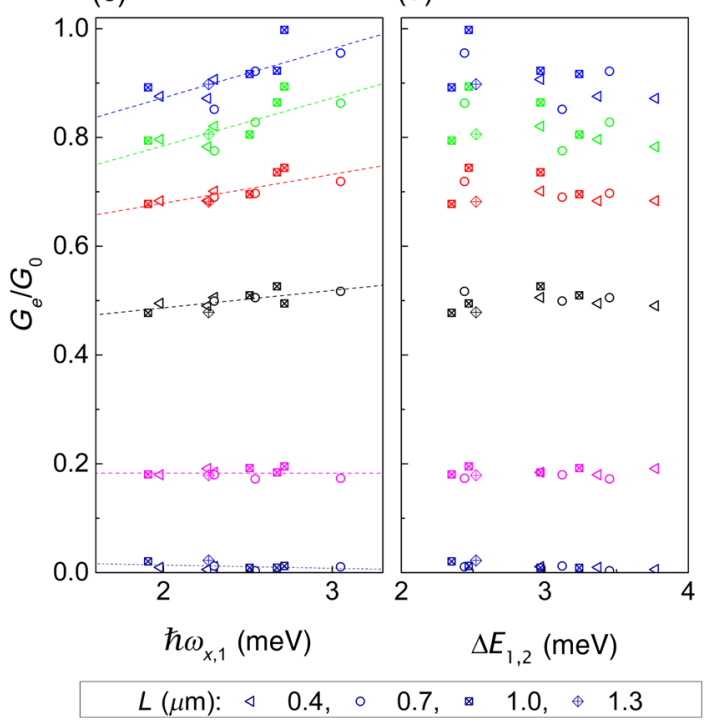

FIG. 5. (a) Fitted conductance $G_{f} / G_{0}$ for the first $1 \mathrm{D}$ subband from 11 devices. Data are collapsed onto a universal curve by aligning $G_{f} / G_{0}=0.5$ with $V_{\mathrm{SG}}=0$, then scaling $V_{\mathrm{SG}}$ by $\alpha_{1} e / \hbar \omega_{x, 1}$. (b) Corresponding experimentally measured conductance $G_{e} / G_{0}$, where the data are offset and scaled using the same parameters as (a). For $G_{e} / G_{0}<0.5$, the traces collapse onto a similar curve. Above $0.5 G_{0}$, variations arise due to the differences in the 0.7 anomaly. (c) $G_{e} / G_{0}$ as a function of barrier curvature $\hbar \omega_{x, 1}$ at fixed values of the scaled voltage axis $\kappa$. From bottom to top, $\kappa$ increases from -0.5 to 0.75 in steps of 0.25 [corresponding to vertical dashed lines, left to right, in panel (b)]. The dashed lines show a linear leastsquares fit to $G_{e} / G_{0}$ for each value of $\kappa$, as a guide to eye. (d) Conductance $G_{e} / G_{0}$ as a function of spacing between the first and second 1D subbands $\Delta E_{1,2}$. In panels (c),(d), data points for each length split gate are indicated by the symbols defined in the legend.

0.7 anomaly (a spread in $G_{e} / G_{0}>1$ arises due to the different $\left.\Delta E_{1,2}\right)$. The variation in the 0.7 anomaly between traces is related to the varying barrier curvature from device to device.

In order to compare the 0.7 anomaly between devices, $G_{e} / G_{0}$ is plotted as a function of $\hbar \omega_{x, 1}$ in Fig. 5(c), for six fixed values of $\kappa$. From bottom to top, $\kappa$ increases from -0.5 to 0.75 in steps of 0.25 [corresponding to the vertical lines in Fig. 5(b)]. For $\kappa=-0.5,-0.25$, and $0, G_{e} / G_{0}$ is independent of $\hbar \omega_{x, 1}$. This is expected since $G_{e} / G_{0}$ is 
below or close to 0.5 . Unique symbols are used to represent data from split gates of each length. The dashed lines are linear fits as a guide to the eye [48].

In the subopen regime, $G_{e} / G_{0}$ reduces with decreasing $\hbar \omega_{x, 1}$. This is consistent with our previous work [28]. However, in Ref. [28], an array of identical-length split gates was measured in which differences in the electrostatic profile between devices arose only from variations in the background potential. In Fig. 5(c), data from split gates of different lengths all follow the same trend line for a given $\kappa$. This implies that the profile of the longitudinal barrierrather than the gate length-is the most significant factor governing the conductance value of the 0.7 anomaly.

The data in Fig. 5 are presented in a similar way as Fig. 2 of Ref. [28] for ease of comparison. We emphasize that these are entirely different data sets from separate devices. The similarity arises because the same technique developed in Ref. [28] to analyze the 0.7 anomaly is applied here.

The importance of device-specific confining potential has been highlighted in Ref. [49], which summarizes numerous studies of the 0.7 anomaly as a function of carrier density. The conductance of the 0.7 anomaly has been seen to both increase and decrease as a function of carrier density. These conflicting trends likely arise due to the extreme sensitivity of the 0.7 anomaly to differences in the electrostatic potential between devices [49].

The trend in Fig. 5(c) is also consistent with the splitgate-length dependence reported in Ref. [7]. The 0.7 anomaly occurred at lower values as the gate length increased (three devices were measured, with lengths of $0,0.5$ and $2 \mu \mathrm{m}$ ). A stronger link between the longitudinal profile and gate length may exist for devices measured in Ref. [7] because they are fabricated on an undoped heterostructure [50], where the absence of dopants may lead to smaller variations in the background potential.

Calculations of the conductance transition between zero and $G_{0}$ using the inelastic scattering model plus the local density-of-states enhancement [22,23] predict a lowering of the conductance in the subopen regime as $\hbar \omega_{x}$ decreases. These calculations are shown in Fig. S14(b) in the Supplemental Material of Ref. [23] and are in agreement with our data. Unfortunately, our data do not allow us to distinguish between theories for the occurrence of the 0.7 anomaly since the same trend is predicted in both the spontaneous spin polarization [51] and Kondo scenarios [52], as discussed in Ref. [28]. However, the inelastic scattering scenario is the only theory for which detailed calculations are performed as a function of $\hbar \omega_{x}$.

Figure 5(d) shows $G_{e} / G_{0}$ as a function of 1D subband spacing $\Delta E_{1,2}$. There is a slight reduction of $G_{e}$ with increasing $\Delta E_{1,2}$ in the subopen regime, although the trend is weak. As seen in Fig. 2(b), a larger $\Delta E_{1,2}$ occurs for devices with a more negative pinch-off voltage. This suggests a possible explanation for the trend in Fig. 5(d): the strength of the transverse confinement is stronger for the devices with larger $\Delta E_{1,2}$, leading to an increase of the strength of electron interactions, thus, affecting the conductance value of the 0.7 anomaly. This relationship may be understood within the framework of the inelastic scattering model, which makes predictions of the effect of the electron interaction strength on the conductance of the 0.7 anomaly. For example, Fig. S14(c) of the Supplemental Material for Ref. [23] shows a reduction of the conductance in the subopen regime with increasing interaction strength for a constant barrier curvature. As a final point of interest, the trends in Figs. 5(c) and 5(d) do not depend on the split-gate length, compatible with the inelastic scattering model which is based purely on the shape of the potential barrier.

\section{CONCLUSION}

We have systematically studied the effect of changing split-gate size on device behavior, using a multiplexing technique which allows many nanostructure devices to be compared on a single cooldown. Multiple devices of each size are measured, providing statistical information on the variance of conductance properties between individual designs. In total, we measure 95 split gates with seven different length and width combinations. Increasing the gate length and/or reducing the width moves the average voltage required to define a $1 \mathrm{D}$ channel and the pinch-off voltage closer to zero. The 1D subband spacing also reduces for devices with longer gates. Further, the percentage of devices displaying accurate quantization of conductance reduces dramatically as the area of the channel increases.

The electrostatic environment in the 2DEG is very influential on the 1D potential profile. The significance of the background potential is highlighted by three key results reported here. First, the spread in values of the 1D subband spacing and the 1D barrier curvature for a given gate length are as large as the overall variation in the average values of these properties over the range of gate lengths measured. Second, the lever arm $\alpha$, which depends on the coupling between the gate and the 1D channel, does not continue to increase as a function of gate length beyond $L=0.7 \mu \mathrm{m}$, a deviation from the expected result. Third, the curvature of the longitudinal potential barrier estimated using a saddle-point model is not strongly related to gate length.

These results imply that (i) gate size cannot be relied upon as a good indicator of the length of the 1D channel, and (ii) the background disorder potential is at least as significant as gate size in determining the potential landscape in the $1 \mathrm{D}$ channel.

The 0.7 anomaly is compared between split gates of different lengths. The conductance value of the 0.7 anomaly reduces as the barrier curvature becomes shallower rather than depending specifically on the split-gate length. The particular confining potential in each device and, principally, the barrier curvature, may be the primary 
factor governing the conductance of the 0.7 anomaly at a given temperature and magnetic field.

\section{ACKNOWLEDGMENTS}

This work is supported by the Engineering and Physical Sciences Research Council Grant No. EP/I014268/1. The data set for this article is available at [53]. The authors thank A. R. Hamilton, J. von Delft, S. Ludwig, and E. T. Owen for helpful discussions, and R. D. Hall for $e$-beam exposure.

[1] D. A. Wharam, T. J. Thornton, R. Newbury, M. Pepper, J. Ahmed, J. E. F. Frost, D. G. Hasko, D. A. Ritchie, and G. A. C. Jones, One-dimensional transport and the quantisation of the ballistic resistance, J. Phys. C 21, L209 (1988).

[2] B. J. van Wees, H. van Houten, C. W. J. Beenakker, J. G. Williamson, L. P. Kouwenhoven, D. van der Marel, and C. T. Foxon, Quantized Conductance of Point Contacts in a Two-Dimensional Electron Gas, Phys. Rev. Lett. 60, 848 (1988).

[3] K. J. Thomas, J. T. Nicholls, M. Y. Simmons, M. Pepper, D. R. Mace, and D. A. Ritchie, Possible Spin Polarization in a One-Dimensional Electron Gas, Phys. Rev. Lett. 77, 135 (1996).

[4] T. J. Thornton, M. Pepper, H. Ahmed, D. Andrews, and G. J. Davies, One-Dimensional Conduction in the 2D Electron Gas of a GaAs-AlGaAs Heterojunction, Phys. Rev. Lett. 56, 1198 (1986).

[5] H.-M. Lee, K. Murakia, E. Y. Chang, and Y. Hirayama, Electronic transport characteristics in a one-dimensional constriction defined by a triple-gate structure, J. Appl. Phys. 100, 043701 (2006).

[6] E. J. Koop, A. I. Lerescu, J. Liu, B. J. van Wees, D. Reuter, A. D. Wieck, and C. H. van der Wal, The influence of device geometry on many-body effects in quantum point contacts: Signatures of the 0.7 anomaly, exchange and Kondo, J. Supercond. Nov. Magn. 20, 433 (2007).

[7] D. J. Reilly, G. R. Facer, A. S. Dzurak, B. E. Kane, R. G. Clark, P. J. Stiles, A. R. Hamilton, J. L. O'Brien, N. E. Lumpkin, L. N. Pfeiffer, and K. W. West, Many-body spin-related phenomena in ultra low-disorder quantum wires, Phys. Rev. B 63, 121311 (2001).

[8] S. J. Koester, B. Brar, C. R. Bolognesi, E. J. Caine, A. Patlach, E. L. Hu, H. Kroemer, and M. J. Rooks, Length dependence of quantized conductance in ballistic constrictions fabricated on InAs/AlSb quantum wells, Phys. Rev. B 53, 13063 (1996).

[9] K. J. Thomas, D. L. Sawkey, M. Pepper, W. R. Tribe, I. Farrer, M. Y. Simmons, and D. A. Ritchie, Unusual conductance collapse in one-dimensional quantum structures, J. Phys. Condens. Matter 16, L279 (2004).

[10] M. J. Iqbal, R. Levy, E. J. Koop, J. B. Dekker, J. P. de Jong, J. H. M. van der Velde, D. Reuter, A. D. Wieck, R. Aguado, Y. Meir, and C. H. van der Wal, Odd and even Kondo effects from emergent localization in quantum point contacts, Nature (London) 501, 79 (2013).
[11] M. J. Iqbal, J. P. de Jong, D. Reuter, A. D. Wieck, and C. H. van der Wal, Split-gate quantum point contacts with tunable channel length, J. Appl. Phys. 113, 024507 (2013).

[12] J. Heyder, F. Bauer, E. Schubert, D. Borowsky, D. Schuh, W. Wegscheider, J. von Delft, and S. Ludwig, Relation between the 0.7 anomaly and the Kondo effect: Geometric crossover between a quantum point contact and a Kondo quantum dot, Phys. Rev. B 92, 195401 (2015).

[13] G. Timp, R. Behringer, S. Sampere, J. E. Cunningham, and R. E. Howard, in Proceedings of the International Symposium on Nanostructure Physics and Fabrication, edited by M. A. Reed and W. P. Kirk (Academic, New York, 1989), p. 331.

[14] K. J. Thomas, J. T. Nicholls, N. J. Appleyard, M. Y. Simmons, M. Pepper, D. R. Mace, W. R. Tribe, and D. A. Ritchie, Interaction effects in a one-dimensional constriction, Phys. Rev. B 58, 4846 (1998).

[15] A. P. Micolich, What lurks below the last plateau: Experimental studies of the $0.7 \times 2 e^{2} / h$ conductance anomaly in one-dimensional systems, J. Phys. Condens. Matter 23, 443201 (2011).

[16] C.-K. Wang and K.-F. Berggren, Spin splitting of subbands in quasi-one-dimensional electron quantum channels, Phys. Rev. B 54, R14257 (1996).

[17] S. M. Cronenwett, H. J. Lynch, D. Goldhaber-Gordon, L. P. Kouwenhoven, C. M. Marcus, K. Hirose, N. S. Wingreen, and V. Umansky, Low-Temperature Fate of the 0.7 Structure in a Point Contact: A Kondo-Like Correlated State in an Open System, Phys. Rev. Lett. 88, 226805 (2002).

[18] Y. Meir, K. Hirose, and N. S. Wingreen, Kondo Model for the "0.7 Anomaly", in Transport through a Quantum Point Contact, Phys. Rev. Lett. 89, 196802 (2002).

[19] T. Rejec and Y. Meir, Magnetic impurity formation in quantum point contacts, Nature (London) 442, 900 (2006).

[20] K. A. Matveev, Conductance of a quantum wire at low electron density, Phys. Rev. B 70, 245319 (2004).

[21] B. Brun, F. Martins, S. Faniel, B. Hackens, G. Bachelier, A. Cavanna, C. Ulysse, A. Ouerghi, U. Gennser, D. Mailly, S. Huant, V. Bayot, M. Sanquer, and H. Sellier, Wigner and Kondo physics in quantum point contacts revealed by scanning gate microscopy, Nat. Commun. 5, 4290 (2014).

[22] C. Sloggett, A. I. Milstein, and O. P. Sushkov, Correlated electron current and temperature dependence of the conductance of a quantum point contact, Eur. Phys. J. B 61, 427 (2008).

[23] F. Bauer, J. Heyder, E. Schubert, D. Borowsky, D. Taubert, B. Bruognolo, D. Schuh, W. Wegscheider, J. von Delft, and S. Ludwig, Microscopic origin of the 0.7-anomaly, in quantum point contacts, Nature (London) 501, 73 (2013).

[24] H. Al-Taie, L. W. Smith, B. Xu, P. See, J. P. Griffiths, H. E. Beere, G. A. C. Jones, D. A. Ritchie, M. J. Kelly, and C. G. Smith, Cryogenic on-chip multiplexer for the study of quantum transport in 256 split-gate devices, Appl. Phys. Lett. 102, 243102 (2013).

[25] L. W. Smith, H. Al-Taie, F. Sfigakis, P. See, A. A. J. Lesage, B. Xu, J. P. Griffiths, H. E. Beere, G. A. C. Jones, D. A. Ritchie, M. J. Kelly, and C. G. Smith, Statistical study of conductance properties in one-dimensional quantum wires focusing on the 0.7 anomaly, Phys. Rev. B 90, 045426 (2014). 
[26] H. Al-Taie, L. W. Smith, A. A. J. Lesage, P. See, J. P. Griffiths, H. E. Beere, G. A. C. Jones, D. A. Ritchie, M. J. Kelly, and C. G. Smith, Spatial mapping and statistical reproducibility of an array of 256 one-dimensional quantum wires, J. Appl. Phys. 118, 075703 (2015).

[27] A. A. J. Lesage, L. W. Smith, H. Al-Taie, P. See, J. P. Griffiths, I. Farrer, G. A. C. Jones, D. A. Ritchie, M. J. Kelly, and C. G. Smith, Assisted extraction of the energy level spacings and lever arms in direct current bias measurements of one-dimensional quantum wires, using an image recognition routine, J. Appl. Phys. 117, 015704 (2015).

[28] L. W. Smith, H. Al-Taie, A. A. J. Lesage, F. Sfigakis, P. See, J. P. Griffiths, H. E. Beere, G. A. C. Jones, D. A. Ritchie, A. R. Hamilton, M. J. Kelly, and C. G. Smith, Dependence of the 0.7 anomaly on the curvature of the potential barrier in quantum wires, Phys. Rev. B 91, 235402 (2015).

[29] R. K. Puddy, L. W Smith, H. Al-Taie, C. H. Chong, I. Farrer, J. P. Griffiths, D. A. Ritchie, M. J. Kelly, M. Pepper, and C. G. Smith, Multiplexed charge-locking device for large arrays of quantum devices, Appl. Phys. Lett. 107, 143501 (2015).

[30] J. H. Davies, I. A. Larkin, and E. V. Sukhorukov, Modeling the patterned two-dimensional electron gas: Electrostatics, J. Appl. Phys. 77, 4504 (1995).

[31] N. K. Patel, J. T. Nicholls, L. Martín-Moreno, M. Pepper, J. E. F Frost, D. A. Ritchie, and G. A. C. Jones, Evolution of half plateaus as a function of electric field in a ballistic quasi-one-dimensional constriction, Phys. Rev. B 44, 13549 (1991).

[32] A. Srinivasan, L. A. Yeoh, O. Klochan, T. P. Martin, J. C. H. Chen, A. P. Micolich, A. R. Hamilton, D. Reuter, and A. D. Wieck, Using a tunable quantum wire to measure the large out-of-plane spin splitting of quasi two-dimensional holes in a GaAs nanostructure, Nano Lett. 13, 148 (2013).

[33] K. J. Thomas, M. Y. Simmons, J. T. Nicholls, D. R. Mace, M. Pepper, and D. A. Ritchie, Ballistic transport in onedimensional constrictions formed in deep two-dimensional electron gases, Appl. Phys. Lett. 67, 109 (1995).

[34] The error associated with the mean value is given by the square root of the averaged square of individual errors divided by the number of data points.

[35] M. Büttiker, Quantized transmission of a saddle-point constriction, Phys. Rev. B 41, 7906 (1990).

[36] J. A. Nixon, J. H. Davies, and H. U. Baranger, Breakdown of quantized conductance in point contacts calculated using realistic potentials, Phys. Rev. B 43, 12638 (1991).

[37] G. Vionnet and O. P. Sushkov, Enhancement Mechanism of the Electron $g$-Factor in Quantum Point Contacts, Phys. Rev. Lett. 116, 126801 (2016).

[38] A. M. Lunde, A. De Martino, A. Schulz, R. Egger, and K. Flensberg, Electron-electron interaction effects in quantum point contacts, New J. Phys. 11, 023031 (2009).

[39] C.-T. Liang, I. M. Castleton, J. E. F. Frost, C. H. W. Barnes, C. G. Smith, C. J. B. Ford, D. A. Ritchie, and M. Pepper, Resonant transmission through an open quantum dot, Phys. Rev. B 55, 6723 (1997).
[40] F. Sfigakis, C. J. B. Ford, M. Pepper, M. Kataoka, D. A. Ritchie, and M. Y. Simmons, Kondo Effect from a Tunable Bound State within a Quantum Wire, Phys. Rev. Lett. 100, 026807 (2008).

[41] J. J. Harris, C. T. Foxon, K. W. J. Barnham, D. Lacklison, J. Hewett, and C. White, Two-dimensional electron gas structures with mobilities in excess of $3 \times 10^{6} \mathrm{~cm}^{2} \mathrm{~V}^{-1} \mathrm{~s}^{-1}$, J. Appl. Phys. 61, 1219 (1987).

[42] C. T. Foxon, J. J. Harris, D. Hilton, J. Hewett, and C. Roberts, Optimisation of (Al, Ga)As/GaAs twodimensional electron gas structures for low carrier densities and ultrahigh mobilities at low temperatures, Semicond. Sci. Technol. 4, 582 (1989).

[43] B. E. Kane, L. N. Pfeiffer, K. W. West, and C. K. Harnett, Variable density high mobility two-dimensional electron and hole gases in a gated $\mathrm{GaAs} / \mathrm{Al}_{x} \mathrm{Ga}_{1-x} \mathrm{As}$ heterostructure, Appl. Phys. Lett. 63, 2132 (1993).

[44] F. Sfigakis, K. Das Gupta, S. Sarkozy, I. Farrer, D. A. Ritchie, M. Pepper, and G. A. C. Jones, Benefits of using undoped GaAs/AlGaAs heterostructures: A case study of the zero-bias bias anomaly in quantum wires, Physica (Amsterdam) 42E, 1200 (2010).

[45] S. Sarkozy, F. Sfigakis, K. Das Gupta, I. Farrer, D. A. Ritchie, G. A. C. Jones, and M. Pepper, Zero-bias anomaly in quantum wires, Phys. Rev. B 79, 161307(R) (2009).

[46] A. M. See, I. Pilgrim, B. C. Scannell, R. D. Montgomery, O. Klochan, A. M. Burke, M. Aagesen, P. E. Lindelof, I. Farrer, D. A. Ritchie, R. P. Taylor, A. R. Hamilton, and A. P. Micolich, Impact of Small-Angle Scattering on Ballistic Transport in Quantum Dots, Phys. Rev. Lett. 108, 196807 (2012).

[47] A. M. See, A. R. Hamilton, A. P. Micolich, M. Aagesen, and P. E. Lindelof, Using light and heat to controllably switch and reset disorder configuration in nanoscale devices, Phys. Rev. B 91, 085417 (2015).

[48] A linear fit is plotted to highlight the upward trend. This is simply a guide to the eye. The range of $\hbar \omega_{x, 1}$ is too small to determine the exact nature of the relationship between $G_{e}$ and $\hbar \omega_{x, 1}$.

[49] A. M. Burke, O. Klochan, I. Farrer, D. A. Ritchie, A. R. Hamilton, and A. P. Micolich, Extreme sensitivity of the spin-splitting and 0.7 anomaly to confining potential in onedimensional nanoelectronic devices, Nano Lett. 12, 4495 (2012).

[50] B. E. Kane, G. R. Facer, A. S. Dzurak, N. E. Lumpkin, R. G. Clark, L. N. Pfeiffer, and K. W. West, Quantized conductance in quantum wires with gate-controlled width and electron density, Appl. Phys. Lett. 72, 3506 (1998).

[51] P. Jaksch, I. Yakimenko, and K.-F. Berggren, From quantum point contacts to quantum wires: Density-functional calculations with exchange and correlation effects, Phys. Rev. B 74, 235320 (2006).

[52] K. Hirose, Y. Meir, and N. S. Wingreen, Local Moment Formation in Quantum Point Contacts, Phys. Rev. Lett. 90, 026804 (2003).

[53] www.repository.cam.ac.uk/handle/1810/249174. 